\title{
Zur Kenntniss der Acetylproducte des Triamidophenols
}

von

\section{Karl Oettinger.}

Aus dem I. chemischen Laboratorium der k. k. Universität in Wren.

(Vorgelegt in der Sitzung am 7. März 1895.)

Wie ich in der vorhergehenden Mittheilung erwähnte, habe ich das Verhalten des salzsauren Triamidophenols gegen Essigsäureanhydrid untersucht. Da ich hiebei zu Resultaten gelangt bin, welche von jenen, die E. Bamberger ${ }^{1}$ erhalten hat, etwas abweichen, so erlaube ich mir in den folgenden Blättern über meine Erfahrungen kurz zu berichten.

B a mberger erhielt bei Einwirkung von Essigsäureanhydrid und essigsaurem Natrium auf das trockene salzsaure Triamidophenol ein Triacetylproduct. Ich erhielt hingegen als Hauptproduct ein Hexaacetyltriamidophenol, daneben geringe Mengen eines Tetra- und Triacetylderivates.

Die Darstellung und Reinigung dieser Verbindungen wurde in folgender Weise bewerkstelligt.

Das salzsaure Triamidophenol wurde vorerst im Vacuumexsiccator bis zur Gewichtsconstanz getrocknet. Hierauf wurden je $50 \mathrm{~g}$ desselben mit der zehnfachen Menge Essigsäuı eanhydrids auf $140-150^{\circ} \mathrm{C}$. im Ölbade in einer Retorte erhitzt. Dabei tritt anfänglich eine lebhafte Entwicklung von Chloracetyl ein, welche

1 Ber. 16, 2400. 
sich allmälig mässigt und nach ungefähr acht Stunden ihr Ende erreicht hat. Nunmehr wird der Überschuss des Essigsäureanhydrids im Vacuum abdestillirt. Es hinterbleibt ein zähes, dunkelbraun gefärbtes Product, welches nach dem Erkalten zu einer asphaltartigen, glänzenden Masse erstarrt. Dieselbe wird zerkleinert, mit einer geringen Menge Wasser übergossen und längere Zeit sich selbst überlassen.

Allmälig tritt die Abscheidung eines gelblichweissen, körnigen Pulvers (a) ein, während ein Theil mit braungelber Farbe in Lösung geht. Die Ausscheidung (a) wurde durch Absaugen gewonnen. Die Lösung, sowie die Waschwässer lieferten nach dem Einengen im Vacuum beim Stehen eine krystallinische Ausscheidung $(b)$. Die Mutterlaugen liefern noch kleine Quantitäten desselben und trocknen endlich $\mathrm{zu}$ einer schmierigen Masse ein, aus welcher keine charakteristischen Substanzen mehr isolirt werden konnten.

\section{Untersuchung von $a$.}

Die Reinigung von $a$ wurde durch wiederholtes Umkrystallisiren aus Alkohol bewerstelligt. Derselbe lost die Verbindung sehr leicht auf und scheidet sie in kleinen, glänzenden Krystallen wieder $a b$, die prismatischen Habitus besitzen und welche zumeist zu lockeren Drusen vereint sind. Der Schmelzpunkt der so gereinigten Substanz liegt bei $184^{\circ} \mathrm{C}$. (uncorr.). Dabei tritt keine Zersetzung ein.

Das Product ist auch in heissem Wasser und Eisessig leicht löslich, Benzol hingegen nimmt es nur in geringen Quantitäten beim Erhitzen auf. Die Verbindung ist ziemlich unempfindlich, was daraus hervorgeht, dass dieselbe weder in Lösung noch für sich an der Luft eine Veränderung erleidet. Auch verträgt sie höhere Temperatur.

Da die Procentgehalte der verschiedenen aus dem Triamidophenol zu gewärtigenden Verbindungen in ihrem C-, Nund $\mathrm{H}$-Gehalt, wie die folgende Tabelle zeigt, ziemlich nahe aneinanderliegen, so mussten directe Acetylbestimmungen vorgenommen werden, um die Anzahl der eingetretenen Acetylreste sicher zu stellen. 


\begin{tabular}{|c|c|c|c|c|}
\hline & Procent $\mathrm{C}$ & Procent $\mathrm{H}$ & Procent $\mathrm{N}$ & $\begin{array}{c}\text { Procent } \\
\mathrm{C}_{2} \mathrm{H}_{3} \mathrm{O}\end{array}$ \\
\hline Monoacetylproduct..... & $53 \cdot 04$ & $6 \cdot 08$ & $23 \cdot 20$ & $23 \cdot 75$ \\
\hline Diacetylproduct .. & $53 \cdot 81$ & $5 \cdot 83$ & $18 \cdot 83$ & $38 \cdot 56$ \\
\hline Triacetylproduct....... & $54 \cdot 34$ & $5 \cdot 66$ & $15 \cdot 85 \vdots$ & $48 \cdot 69$ \\
\hline Tetraacetylproduct..... & $54 \cdot 72$ & $5 \cdot 54$ & $13 \cdot 68 r$ & $56 \cdot 02$ \\
\hline Pentaacetylproduct.... & $55 \cdot 01$ & $5 \cdot 44$ & $12 \cdot 03$ & $61 \cdot 60$ \\
\hline Hexaacetylproduct. & $55 \cdot 24$ & $5 \cdot 37$ & $10 \cdot 7 \pm$ & $65 \cdot 99$ \\
\hline Heptaacetylproduct .... & $55 \cdot 43$ & $5 \cdot 31$ & $9 \cdot 70$ & $69 \cdot 51$ \\
\hline
\end{tabular}

Zur Analyse habe ich die Substanz bei $100^{\circ}$ zur Gewichtsconstanz getrocknet und erhielt dabei Werthe, welche mit Bestimmtheit darauf hinweisen, dass hier ein Hexaacetyltriamidophenol

$$
\left.\mathrm{C}_{6} \mathrm{H}_{2}\left(\mathrm{OC}_{2} \mathrm{H}_{3} \mathrm{O}\right)\left(\mathrm{NHC}_{2} \mathrm{H}_{3} \mathrm{O}\right)\left(\mathrm{N}_{(} \mathrm{C}_{2} \mathrm{H}_{3} \mathrm{O}\right)_{2}\right)_{2}
$$

vorliegt. Dieselben ergaben:

I. $0 \cdot 2122 g$ Substanz gaben $0 \cdot 4298 g \mathrm{CO}_{2}$ und $0 \cdot 1055 g \mathrm{H}_{2} \mathrm{O}$.

II. $0 \cdot 1954 \mathrm{~g}$ Substanz gaben $19.4 \mathrm{~cm}^{3} \mathrm{~N}$ bei $20.5^{\circ} \mathrm{C}$. und $751 \cdot 3 \mathrm{~mm}$.

III. $0 \cdot 2235 g$ Substanz gaben $0 \cdot 4547 g \mathrm{CO}_{2}$ und $0 \cdot 1025 g \mathrm{H}_{2} \mathrm{O}$. IV. $0.2413 \mathrm{~g}$ Substanz gaben $23 \mathrm{~cm}^{3} \mathrm{~N}$ bei $16^{\circ}$ und $757.5 \mathrm{~mm}$.

V. $0.8082 \mathrm{~g}$ Substanz gaben $0.7316 \mathrm{~g}$ Essigsäure.

In 100 Theilen:

\begin{tabular}{|c|c|c|c|c|c|}
\hline J. & II. & III. & IV. & v. & Gerechnet \\
\hline С . . . 55.24 & - & $55 \cdot 48$ & - & - & $55 \cdot 21$ \\
\hline H .... 5.52 & - & $5 \cdot 10$ & - & - & $5 \cdot 37$ \\
\hline$N \ldots \ldots-$ & $11 \cdot 19$ & - & $1+11 \cdot 07$ & - & $10 \cdot 74$ \\
\hline $\mathrm{C}_{2} \mathrm{H}_{3} \mathrm{O} \ldots$ & - & - & $\ldots$ & $64 \cdot 87$ & $65 \cdot 99$ \\
\hline
\end{tabular}

Das Hexaacetyltriamidophenol gibt mit Eisenchlorid keine Farbreaction. Beim längeren Schütteln mit verdünnter Kalilauge löst es sich allmälig auf. Säuert man diese Lösung mit Essigsäure an, so fällt, vorausgesetzt, dass die Flüssigkeit entsprechend concentrirt war, ein weisser krystallinischer Niederschlag aus, der jedoch wie der Schmelzpunkt (263) zeigt, 
nicht mehr die ursprüngliche Substanz ist. Offenbar hat hier eine Abspaltung von Essigsäure stattgefunden. Wird diese Ausscheidung aus Alkohol umkrystallisirt, so erhöht sich der Schmelzpunkt derselben auf $279^{\circ}$ (uncorr.) Demnach dürfte durch die Zersetzung der ursprünglichen Verbindung das von Bamberger beschriebene Triacetyltriamidophenol entstanden sein, welc'.es nach seinen Angaben den Schmelzpunkt $263^{\circ}$ besitzt. Dasselbe ist in Kalilauge sehr leicht löslich und daraus. folgert $\mathrm{B}$ am $\mathrm{m}$ erger, dass die Hydroxylgruppe intact geblieben ist. Da das Hexaacetylproduct sich in Kalilauge nicht gleich, sondern ersi nach einiger Zeit löst, so kann man wohl annehmen, dass in dic em Producte auch ein Eintritt von Acetyl in die Hydroxylgruppe stattgefunden hat.

Das vorhin erwähnte Zersetzungsproduct vom Schmelzpunkt $279^{\circ}$ i. $t$ auch der Hauptbestandtheil der Krystallfraction, die ich mit $(b)$ bezeichnet habe.

\section{Untersuchung von $b$.}

Dieses ist ein Gemisch von Tetraacetyl- und von Triacetyltriamidophenol. Die Trennung der beiden Körper kann durch systematische, fractionirte Krystallisation aus Wasser vorgenommen werden. Die sich erst ausscheidenden Producte enthalten vorweg das Triacetyltriamidophenol, während in den Laugen das Tetraacetylproduct zurückbleibt und nach dem Abdestilliren aus denselben erhalten wird.

\section{Triacetyltriamidophenol$$
\mathrm{C}_{6} \mathrm{H}_{2} \mathrm{OH}\left(\mathrm{NHC}_{2} \mathrm{H}_{3} \mathrm{O}\right)_{3} \text {. }
$$

Bildet nach wiederholtem Umkrystallisiren aus siedendem Wasser (eventuell Entfärben mit Thierkohle) ein Aggregat von farblosen kleinen Krystallen, die unter dem Mikroskop betrachtet als scharf ausgebildete Tafeln erscheinen, welche dem monoklinen System angehören dürften.

Die vollkommen reine, trockene Substanz zeigt den Schmelzpunkt von $279^{\circ}$ (uncorr.); bei ungefähr $260^{\circ}$ tritt Braunfärbung ein. Minder sorgfältig gereinigte oder nicht vollständig trockene Partien zeigen einen tieferen, um $260^{\circ}$ liegenden Schmelzpunkt. 
Wasser und Alkohol nehmen die Substanz in der Hitze auf. Die anderen Lösungsmittel, wie Äther, Benzol, Chloroform lösen sie selbst beim Kochen nur in sehr geringen Mengen.

Die Analysen ergaben Werthe, die, wie aus der obenstehenden Tabelle ersichtlich, mit den für das Triacetylproduct gerechneten völlig übereinstimmen.

I. $0.3106 \mathrm{~g}$ Substanz gaben $0.6168 \mathrm{~g} \mathrm{CO}_{2}$ und $0.1572 \mathrm{~g} \mathrm{H}_{2} \mathrm{O}$.

II. $0 \cdot 2440 \mathrm{~g}$ Substanz gaben $0 \cdot 4878 g \mathrm{CO}_{2}$ und $0 \cdot 1311 \mathrm{~g} \mathrm{H}_{2} \mathrm{O}$. III. $0 \cdot 2411 \mathrm{~g}$ Substanz gaben $33.2 \mathrm{~cm}^{3} \mathrm{~N}$ bei $17^{\circ}$ und $753.5 \mathrm{~mm}$. IV. $0.2884 \mathrm{~g}$ Substanz gaben $41.1 \mathrm{~cm}^{3} \mathrm{~N}$ bei $23^{\circ}$ und $747 \mathrm{~mm}$. V. $0.5132 \mathrm{~g}$ Substanz gaben $0.3366 \mathrm{~g}$ Essigsäure.

In 100 Theilen:

\begin{tabular}{|c|c|c|c|c|c|}
\hline I. & II. & III. & IV. & v. & Gerechnet \\
\hline$C \ldots .54 \cdot 16$ & $54 \cdot 52$ & - & - & - & $54 \cdot 34$ \\
\hline H .... $5 \cdot 62$ & $5 \cdot 97$ & - & - & - & $5 \cdot 66$ \\
\hline$N \ldots \ldots-$ & - & $15 \cdot 83$ & $15 \cdot 77$ & - & $15 \cdot 85$ \\
\hline $\mathrm{C}_{2} \mathrm{H}_{3} \mathrm{O} \ldots-$ & - & - & - & $47 \cdot 01$ & $48 \cdot 69$ \\
\hline
\end{tabular}

Das Triacetylproduct ist in Kalilauge leicht löslich, fällt mit Essigsäure wieder unverändert aus und dürfte daher und auch mit Rücksicht auf seine sonstigen Löslichkeitsverhältnisse identisch sein mit der Verbindung, welche Bamberger beschrieben hat, zumal dasselbe in wässeriger Lösung und auch durch Erhitzen mit verdünnten Säuren allmälig zersetzt wird und Triamidophenol rückbildet. Auch entsteht in der wässerigen Lösung durch Zugabe von Eisenchlorid nach einiger Zeit die Ausscheidung von glänzenden goldgelben Kryställchen, die den Schmelzpunkt von $264^{\circ}$ (uncorr.) zeigen. Dieses Verhalten ist für das Bamberger'sche Triacetylproduct charakteristisch.

\section{Tetraacetyltriamidophenol$$
\mathrm{C}_{6} \mathrm{H}_{2}\left(\mathrm{OC}_{2} \mathrm{H}_{3} \mathrm{O}\right)\left(\mathrm{NHC}_{2} \mathrm{H}_{3} \mathrm{O}\right)_{3} \text {. }
$$

Bildet nach wiederholtem Umkrystallisiren aus Wasser unter Anwendung von etwas Thierhohle ein sandiges weisses Pulver, welches aus farblosen, ziemlich glänzenden, prismenförmigen Kryställchen besteht. Die Verbindung ist in Alkohol und auch in Wasser wesentlich leichter löslich als das vorhin 
besprochene Triacetylproduct. Gegen die übrigen Lösungsmittel zeigt es ein ähnliches Verhalcen.

Das Tetraacetyltriamidophenol schmilzt nach vorhergehender Braunfürbung unter Zersetzung bei $255^{\circ}$ (uncorr.).

Die Analysen und die Acetylbestimmung führte zu Werthen, welche mit den theoretischen, wie aus der Tabelle $z u$ entnehmen ist, vollkommen übereinstimmen.

I. $0 \cdot 2911 g$ Substanz gaben $0 \cdot 5841 \mathrm{~g} \mathrm{CO}_{2}$ und $0 \cdot 1489 g \mathrm{H}_{2} \mathrm{O}$. II. $0.2389 \mathrm{~g}$ Substanz gaben $29 \mathrm{~cm}^{3} \mathrm{~N}$ bei $18^{\circ}$ und $765.5 \mathrm{~mm}$. III. $0 \cdot 2122 \mathrm{~g}$ Substanz gaben $25.5 \mathrm{~cm}^{3} \mathrm{~N}$ bei $14^{\circ}$ und $756 \mathrm{~mm}$. IV. $1.0517 \mathrm{~g}$ Substanz gaben $0 \cdot 82287 \mathrm{~g}$ Essigsäure.

In 100 Theilen:

$\begin{array}{lccccc} & \text { I. } & \text { II. } & \text { III. } & \text { IV. } & \text { Gerechnet } \\ \mathrm{C} \ldots \ldots \ldots 54 \cdot 72 & - & - & - & 54 \cdot 72 \\ \mathrm{H} \ldots \ldots \ldots \ldots 5 \cdot 68 & - & - & - & 5 \cdot 54 \\ \mathrm{~N} \ldots \ldots \ldots & - & 14 \cdot 08 & 14 \cdot 02 & - & 13 \cdot 68 \\ \mathrm{C}_{2} \mathrm{H}_{3} \mathrm{O} \ldots \ldots & - & - & - & 56 \cdot 07 & 56 \cdot 02\end{array}$

Auch das Tetraacetylproduct löst sich in verdünnter Kalilauge auf; mit Essigsäure angesäuert fällt eine krystallinische Masse, die sich jedoch als Triacetyltriamidophenol $\left(279^{\circ}\right)$ erwies. Demnach findet auch hier wie beim früher besprochenen Hexaacetylderivat Abspaltung von Essigsäure statt.

Bei der Einwirkung von Essigsäureanhydrid auf das salzsaure Triamidophenol wird offenbar nur das Hexaacetyltriamidophenol gebildet, welches durch die Wasserwirkung partielle Zersetzung erfährt und so Tri-, Tetra- und möglicherweise auch andere Acetylproducte liefert. Dass eine derartige Zersetzung wirklich vor sich geht, beweist der Umstand, dass ich aus den Mutterlaugen von $a$, die doch gewiss nicht frei von Hexaacetylproduct waren, nach dem Abdampfen niemals diese Verbindung mehr erhalten konnte. 